\title{
BMJ Open Understanding the low take-up of home-based dialysis through a shared decision-making lens: a qualitative study
}

\author{
Jane Noyes (D) , ${ }^{1}$ Gareth Roberts (D) , ${ }^{2}$ Gail Williams, ${ }^{3}$ James Chess (D) , ${ }^{4}$ \\ Leah Mc Laughlin (D) ${ }^{1}$
}

To cite: Noyes J, Roberts G, Williams $\mathrm{G}$, et al. Understanding the low take-up of homebased dialysis through a shared decision-making lens: a qualitative study. BMJ Open 2021;11:e053937. doi:10.1136/ bmjopen-2021-053937

- Prepublication history and additional supplemental material for this paper are available online. To view these files, please visit the journal online (http://dx.doi.org/10.1136/ bmjopen-2021-053937).

Received 30 May 2021 Accepted 21 October 2021

A) Check for updates

C Author(s) (or their employer(s)) 2021. Re-use permitted under CC BY-NC. No commercial re-use. See rights and permissions. Published by BMJ.

${ }^{1}$ School of Medical and Health Sciences, Bangor University, Bangor, UK

${ }^{2}$ Department of Nephrology, Cardiff and Vale University Health Board, Cardiff, UK ${ }^{3}$ Welsh Clincal Renal Network, NHS Wales, Cardiff, UK

${ }^{4}$ Renal Unit, Swansea Bay University Health Board, Port Talbot, UK

Correspondence to

Dr Jane Noyes;

jane.noyes@bangor.ac.uk

\section{ABSTRACT}

Objectives To explore how people with chronic kidney disease who are pre-dialysis, family members and healthcare professionals together navigate common shared decision-making processes and to assess how this impacts future treatment choice.

Design Coproductive qualitative study, underpinned by the Making Good Decisions in Collaboration shared decisionmodel. Semistructured interviews with a purposive sample from February 2019 - January 2020. Interview data were analysed using framework analysis. Coproduction of logic models/roadmaps and recommendations.

Setting Five Welsh kidney services.

Participants 95 participants (37 patients, 19 family members and 39 professionals); 44 people supported coproduction (18 patients, 8 family members and 18 professionals).

Findings Shared decision-making was too generic and clinically focused and had little impact on people getting onto home dialysis. Preferences of where, when and how to implement shared decision-making varied widely. Apathy experienced by patients, caused by lack of symptoms, denial, social circumstances and health systems issues made future treatment discussions difficult. Families had unmet and unrecognised needs, which significantly influenced patient decisions. Protocols containing treatment hierarchies and standards were understood by professionals but not translated for patients and families. Variation in dialysis treatment was discussed to match individual lifestyles. Patients and professionals were, however, defaulting to the perceived simplest option. It was easy for patients to opt for hospital-based treatments by listing important but easily modifiable factors.

Conclusions Shared decision-making processes need to be individually tailored with more attention on patients who could choose a home therapy but select a different option. There are critical points in the decision-making process where changes could benefit patients. Patients need to be better educated and their preconceived ideas and misconceptions gently challenged. Healthcare professionals need to update their knowledge in order to provide the best advice and guidance. There needs to be more awareness of the costs and benefits of the various treatment options when making decisions.
Strengths and limitations of this study

- Large-scale qualitative study with depth data from patients, family members and professionals.

- New understandings of actions and behaviours following pre-dialysis shared decision-making in a UK healthcare setting.

- Products include coproduced logic models and a road map and recommendations to support implementation of shared decision-making in a complex healthcare system.

- Black and Minority Ethnic (BAME) perspectives, and some underrepresented groups are a noted gap.

- Some findings and the products may not be applicable to substantially different healthcare settings and systems.

\section{INTRODUCTION}

The increasing global prevalence of kidney disease and the burden of dialysis on healthcare systems have driven the need to increase home-based dialysis. ${ }^{1}$ Dialysis in a hospital or satellite unit is collectively called Unit Haemodialysis (UHD) and is undertaken 3 times a week for 4 hour sessions at a time. Homebased therapies have a number of advantages compared with UHD. More frequent and longer dialysis is possible at home and can be associated with extended survival rates. ${ }^{2-6}$ People with chronic kidney disease (CKD) receiving home dialysis experience better quality of life through greater independence and autonomy. ${ }^{78}$ The flexibility of selfmanagement and reduced need to travel to a dialysis unit provides greater opportunities for people to take on occupational and social roles. ${ }^{9}$ Home dialysis is more cost-effective in many circumstances. ${ }^{10-12}$ Those opting for UHD have spoken about their lack of confidence to undertake home-based care. ${ }^{13} 14$

Clinical practice guidance in many highincome countries (eg, the USA, Canada, 
Australia, UK and Europe) state that patients with CKD who may need kidney replacement therapy should be offered support to choose the dialysis modalityincluding home therapies-that best reflects their, and/ or their family's circumstances, needs and values. ${ }^{15}$ Recent updates to UK National Institute for Health and Care Excellence (NICE) guidance suggest that conversations with patients about future treatment options should start at least a year in advance of needing dialysis. ${ }^{16}$ Different dialysis options continue to be made available, supposedly offering more tailored treatments and, therefore, a need for carefully tailored shared decision-making (SDM) in a clinical context. ${ }^{17}$ UK trends show that UHD continues to increase and a huge variation of uptake of home dialysis between centres. ${ }^{18}$

Pre-dialysis education has previously been reported by patients across similar healthcare systems as being overwhelming and biased towards available rather than all options. ${ }^{19}$ Ideally, choosing an option should happen through effective $\mathrm{SDM}^{15}$ as poor decision-making experiences are associated with low treatment satisfaction. ${ }^{20}$ Yet, while an estimated $50 \%$ of all patients are suitable for home peritoneal dialysis (PD) and at least $15 \%$ of patients with haemodialysis could dialyse at home, ${ }^{21}$ international evidence shows that less than a fifth of dialysis takes place in the home. ${ }^{22}$ Barriers to home dialysis have been found to be complex. ${ }^{23}$ Patients have reported insufficient knowledge and education as a barrier to selecting home dialysis. ${ }^{24}$ Practically, home dialysis requires people to have adequate housing including space for supplies and in the case of home haemodialysis (HHD), an affordable and satisfactory supply of water. ${ }^{25}$ Studies also report a complex mix of more intractable social, psychological, economic and health system obstacles to home dialysis. $8142026-28$

Gaining a better understanding of the factors that impact on treatment choices, and, specifically, to better understand why so many patients default to UHD was the aim of the 'Dialysis Options and Choices Study'. This 2-year mixed method coproductive study integrated analysis of electronic renal patient records with content analysis of pre-dialysis patient education programmes, interviews with pre-dialysis patients and their families, interviews with healthcare professionals and health economic analysis of dialysis modalities.

This paper presents findings from the qualitative component of the study examining the factors that impact on pre-dialysis choices made by patients. Using qualitative methods and framed within a model of SDM, we explored what patients and family members actually understand about the disease, the prognosis and the treatment options, how people's values and preferences impact on their choices and the extent to which these were elicited by clinical teams within the SDM process. We also explored the perspective of kidney healthcare professionals. In the context of diverse service provision within a healthcare system, the paper concludes by setting out options to improve healthcare and services towards meeting the policy intent of increased uptake of home therapies.

\section{Methods}

This was a qualitative coproductive study using the framework method ${ }^{30}$ of data collection and analysis and reported using the Consolidated Criteria for Reporting Qualitative Studies checklist ${ }^{31}$ (online supplemental appendix 1). A protocol was developed and published. ${ }^{29}$

\section{THEORETICAL FRAMEWORK}

The study was informed by theory underpinning the Making Good Decisions in Collaboration (MAGIC) model and programme designed to test and identify the best ways to embed SDM into routine primary and secondary care. ${ }^{32} \mathrm{SDM}$ proposes that informed preferences-by which is meant what matters to people with health needs and families-should play a major role in decision-making instead of the assumption that decisions should be guided by scientific consensus about treatment effectiveness. ${ }^{33}$ The MAGIC programme provides a multistage consultation process for decisions-such as dialysis modality-involving a 'three-talk model' for SDM. ${ }^{34}$ The model was adapted for this study to reflect pre-dialysis decision-making (figure 1).

\section{Setting}

The study took place in Wales, a semiautonomous country within the UK with a publicly funded healthcare system. There are five kidney services across the country that are commissioned, monitored and audited by the Welsh Renal Clinical Network. Incidence and prevalence of dialysis is higher in Wales than in other parts of the UK and despite half of patients being suitable for home therapies, only $12.9 \%$ are on PD and only $6.1 \%$ are on HHD with significant variation in patient modality choice across the country. ${ }^{35}$ Wales also has an older and sicker population than the rest of the UK, with a mix of rural and urban settings, pockets of high deprivation with health literacy a recognised issue across all healthcare. ${ }^{36} 37$

\section{ETHICS}

Ethical approval for this study was granted by the Wales Research Ethics Committee (Reference 19/WA/0020).

\section{PATIENT, PUBLIC AND STAKEHOLDER INVOLVEMENT}

The study was funded by Health and Care Research Wales under the Research for Patient and Public Benefit Scheme and a themed call: 'Can co-production lead to more sustainable services'. Elsewhere, we report the outcomes from the coproduction in relation to specific service improvement aspects of the study. ${ }^{23}$

In this paper, we include the patient and public involvement (PPI) and wider stakeholder activity related to the empirical research study. We map the PPI in the study against the UK standards for public involvement ${ }^{38}$ in an 


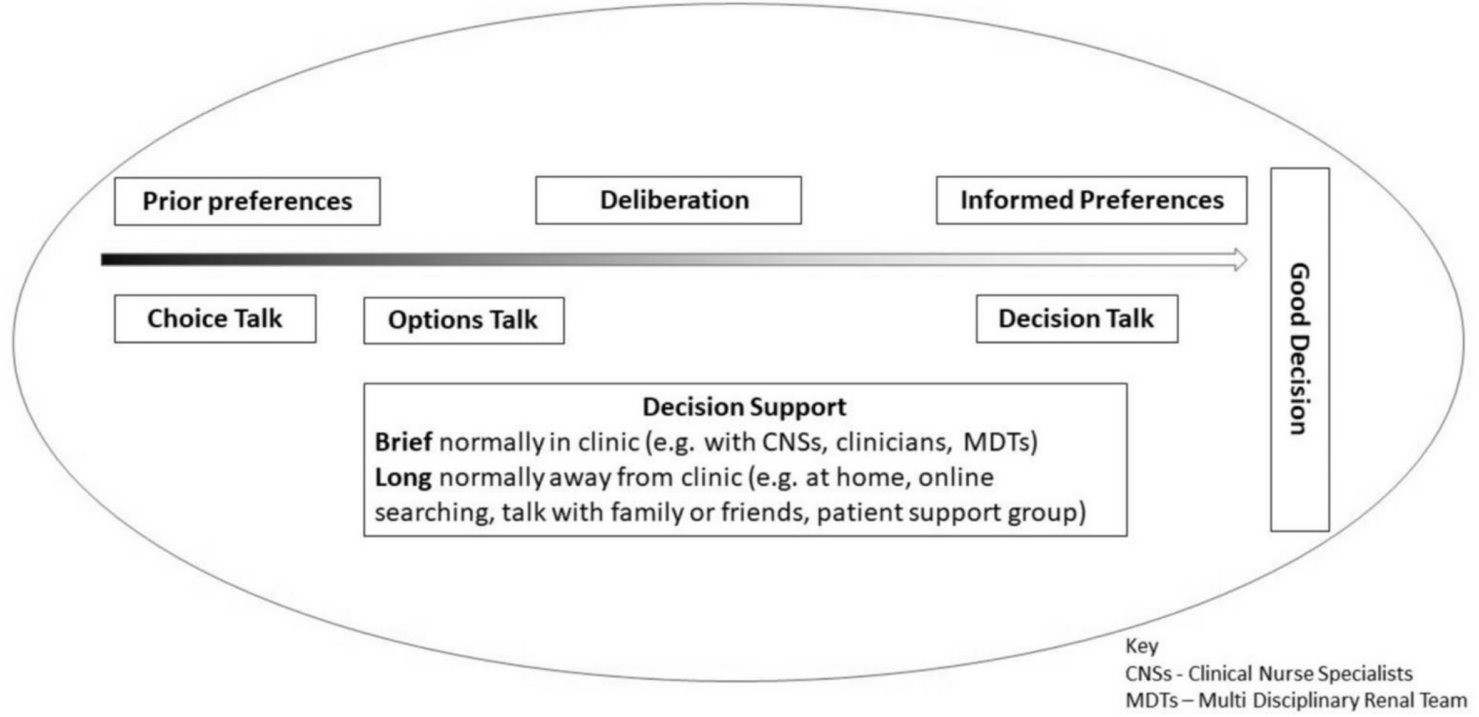

Figure 1 Three-talk model of shared decision-making adapted for pre-dialysis decision making.

online file (online supplemental appendix 2). Three PPI/ stakeholder meetings were specifically curated to present data and gain input from people not directly involved in the study but who had expert perspectives. We hosted these meetings to present and receive input on three key elements (1) pre-dialysis SDM processes, (2) interviews with patients, family members and professionals and (3) developing the logic model of stages, moderators and outcomes of getting onto a home therapy and a road map to service redesign, including practice recommendations (box 1).

\section{Data collection}

Patient and family members' interviews

Any person over 18 within 5 years of pre-dialysis education and their family members were eligible, including those family members who identified as a caregiver. ${ }^{39}$ Participants were purposively sampled to represent a range of decision-making processes (including those who remained 'undecided' post initial education programme visit) in addition to demographics including deprivation, education level, age, gender and kidney service. (table 1) Nurses who deliver pre-dialysis education were tasked with

\section{Box 1 Patient, public and stakeholder involvement} contribution to coproduction of this element

Total numbers: $n=44$; professionals $n=18$, patients $n=18$ and family members $n=8$.

- Pre-dialysis shared decision-making meeting: total $(\mathrm{n}=14)$; professionals $n=8$, patients $n=4$ and family members $n=2$.

- Interview input and feedback: total $(n=30)$; professionals $n=10$, patients $n=14$ and family member $n=6$.

- Developing logic model and road map: total $(n=14)$; professionals $n=5$, patients $n=5$ and family members $n=4$.

- Additional input was provided throughout via emails, face-to-face meetings and informal conversations with patient and public involvements and kidney professionals. identifying potential participants and seeking consent for the research team to contact. Interviews continued until no new themes were coming through and the sample represented a maximum variation. Table 2 and online supplemental appendix 3 provides further details of interview processes with demographics presented in table 1 .

\section{Professional interviews}

Members of the research team who were also healthcare professionals and highly engaged in SDM with patients helped identify and send out initial invitations to key people involved in pre-dialysis decision-making, namely nephrologists and the pre-dialysis nurse specialists. Many nephrologists have specialist training in certain kidney replacement therapies (eg, transplant) and others have specific interests in service improvements such as clinics for people with early-stage CKD sometimes called 'low clearance clinics', or improving awareness and integration of conservative management in the dialysis healthcare teams. We aimed to include a spectrum of Nephrologists representing expertise and interests across the kidney health service. Every specialist pre-dialysis nurse in Wales was invited to interview. We also targeted nurse and service managers to ensure that a whole systems perspective was included. The primary purpose of the professional interviews was to unpack biases and other perceived barriers to people opting for home-based therapies. Table 3 and online supplemental appendix 4 provide further details of professional interviews.

\section{Interviews}

Digital recordings of the interviews were transcribed verbatim by a specialist service and uploaded into NVIVO V.11 (QSR International). Codes were created to link participants with each other and with their respective kidney centre. We used the five-stage framework analysis method to organise and code interview data. ${ }^{40}$ The 'three-talk model' can be viewed from the perspective of 


\begin{tabular}{|c|c|c|c|}
\hline $\begin{array}{l}\text { Total number } \\
\text { of participants }\end{array}$ & $\mathrm{n}=56$ & $\begin{array}{l}\text { Total number } \\
\text { of patients }\end{array}$ & $n=37$ \\
\hline Female & $n=30$ & Female & $\mathrm{n}=17$ \\
\hline \multirow[t]{4}{*}{ Male } & $\mathrm{n}=26$ & Male & $n=20$ \\
\hline & & $\begin{array}{l}\text { Patients chosen } \\
\text { treatment }\end{array}$ & \\
\hline & & UHD & $n=16$ \\
\hline & & HHD & $n=9$ \\
\hline Age group & & CAPD & $n=5$ \\
\hline Under 30 & $n=2$ & APD & $n=4$ \\
\hline $31-39$ & $n=3$ & $\begin{array}{l}\text { Conservative } \\
\text { management }\end{array}$ & $\mathrm{n}=1$ \\
\hline $40-49$ & $n=8$ & Undecided & $n=2$ \\
\hline $50-59$ & $\mathrm{n}=9$ & & \\
\hline $60-69$ & $n=19$ & $\begin{array}{l}\text { Time on } \\
\text { current kidney } \\
\text { replacement } \\
\text { treatment }\end{array}$ & \\
\hline $70-79$ & $\mathrm{n}=14$ & Not started & $\begin{array}{l}\mathrm{n}=23 \text { ( } 1 \\
\text { conservative } \\
\text { management) }\end{array}$ \\
\hline \multirow[t]{2}{*}{$80-89$} & $n=1$ & Under 1 year & $\mathrm{n}=8$ \\
\hline & & $1-3$ years & $n=5$ \\
\hline \multicolumn{4}{|l|}{$\begin{array}{l}\text { Highest } \\
\text { qualification }\end{array}$} \\
\hline None & $\mathrm{n}=9$ & Kidney service & \\
\hline Level 1 & $n=11$ & Cardiff & $n=17$ \\
\hline Level 2 & $\mathrm{n}=7$ & Swansea & $\mathrm{n}=7$ \\
\hline Level 3 & $n=13$ & Bangor & $n=2$ \\
\hline Level 4 & $n=1$ & Glan Clwyd & $\mathrm{n}=5$ \\
\hline $\begin{array}{l}\text { Level } 5 \\
\text { Income }\end{array}$ & $n=5$ & $\begin{array}{l}\text { Wrexham } \\
\text { Total number of } \\
\text { family members }\end{array}$ & $\begin{array}{l}n=6 \\
n=19\end{array}$ \\
\hline $\begin{array}{l}\text { Less than } \\
20 \mathrm{~K}\end{array}$ & $n=21$ & Female & $n=13$ \\
\hline $20 \mathrm{~K}-39 \mathrm{~K}$ & $\mathrm{n}=22$ & Male & $n=6$ \\
\hline $40 \mathrm{~K}-59 \mathrm{~K}$ & $n=13$ & & \\
\hline $\begin{array}{l}\text { More than } \\
60 \mathrm{~K}\end{array}$ & $n=1$ & & \\
\hline \multicolumn{4}{|l|}{ Ethnicity } \\
\hline $\begin{array}{l}\text { Welsh/English/ } \\
\text { Scottish/ } \\
\text { Northern Irish/ } \\
\text { British }\end{array}$ & $\mathrm{n}=56$ & & \\
\hline \multicolumn{4}{|l|}{$\begin{array}{l}\text { Area of } \\
\text { deprivation* }\end{array}$} \\
\hline $\begin{array}{l}\text { 1. Least } \\
\text { deprived }\end{array}$ & $n=12$ & & \\
\hline 2. & $\mathrm{n}=5$ & & \\
\hline
\end{tabular}

Continued

\begin{tabular}{ll|l|} 
Table 1 Continued & \\
\hline $\begin{array}{l}\text { Total number } \\
\text { of participants }\end{array}$ & $\mathrm{n}=56$ & $\begin{array}{l}\text { Total number } \\
\text { of patients } \quad \mathrm{n}=\mathbf{3 7}\end{array}$ \\
\hline 3. & $\mathrm{n}=7$ & \\
4. & $\mathrm{n}=5$ & \\
$\begin{array}{l}\text { 5. Most } \\
\text { deprived }\end{array}$ & $\mathrm{n}=8$ & \\
\hline
\end{tabular}

*Overall score taken from Welsh Index of Multiple deprivation an online resource to estimate deprivation based on income, employment, Health, Education, Access to Services, Community Safety, Physical Environment and housing https://wimd. gov.wales/?_ga=2.206133845.791407745.1630500908977393655.1630500908.

APD, Automated peritoneal dialysis ; CAPD, Continuous ambulatory peritoneal dialysis; HHD, home haemodialysis; UHD, Unit-based dialysis.

the patient, family or professional and was used as the high-level framework to code interview transcripts.

\section{Rigour}

To ensure a satisfactory level of understanding and agreement between coders, four sample transcripts were selected at the start of the process for discussion to ensure consistency. Additional codes of interest were added from a sample of 10 initial interviews, which were read line-byline by two experienced coders and discussed with the multidisciplinary research team at weekly meetings and at wider stakeholder meetings as data were gathered and coding increased.

Discussion of emerging themes, patterns and tendencies began as soon as data became available to share. We used Lincoln and Guber's four dimension criteria (credibility, dependability, confirmability and transferability) to establish rigour. ${ }^{41}$ Examples included a multi-disciplinary team (MDT) of core researchers, PPI involvement in developing data collection tools, purposive sample framework and coproductive analysis and interpretation. Members of the multidisciplinary research team were able to share their expertise and perspectives to help further contextualise data, explain anomalies and advise on ways to fill gaps in the data set. Discrepancies were resolved through whole team discussion. Emerging findings were also presented to PPI/stakeholder groups who had an opportunity to comment and share their expert knowledge (box 1).

\section{Reflexivity}

From a reflexive standpoint, the mixed clinical/academic team believed that home therapy could be an appropriate option for many people who decided against it, and this was clarified in our epidemiological analysis of routinely collected kidney patient data. Wales policy explicitly states that, where appropriate, treatment should be shifted out of hospitals into the communities and towards home and the policy imperative of increasing home dialysis spoke to this agenda. ${ }^{42}$ The team also hypothesised that in spite of 
Table 2 Further details of people with CKD and family members' interview selection and processes

Topic guides

Topic guides were informed by the 'three-talk' model and were piloted during initial interviews with PPI involvement. Topic guides were created for people with CKD, family members as well as options for dialysis and included specific probes to help unpack why patients and family members had chosen a specific treatment over and above another treatment. When both people with CKD and family members were interviewed together, specific questions relevant for the family were posed to them and vice versa to help unpick where views and experiences were different and potentially influencing decisions (online supplemental appendix 3).

Interviewees Interviews were undertaken by male and female researchers with relevant Doctorates who were experienced in interviewing and employed to work on the study. Interviewers had no prior relationship with patients or family members. Some of the professionals were known to one researcher.

Number of interviews 37 interviews covering 37 individual patient cases, with a mix of one to one with patients, family members and joint interviews with patients and their spouse/partner.

Length of interviews Semistructured between $30 \mathrm{~min}$ and $90 \mathrm{~min}$. Participants were interviewed once.

Reasons for decline Most people who were contacted consented to an interview with some declining due to time available to interview, recent bereavement or health issues. We also were contacted by more people than we had capacity to interview and used a theoretical sampling frame to construct a maximum variation sample.

CKD, chronic kidney disease; PPI, patient and public involvement.

expectations that SDM was operating as business as usual (with one kidney service actually involved in the implementation of SDM across the NHS), SDM had yet to be widely adopted in routine clinical encounters. ${ }^{32}$ The team also acknowledged that if following a SDM model, patients came to a treatment decision that they were happy with, then 'SDM' had occurred and the patient had made their choice. Nonetheless, the imperative for this study was to explore decision-making within the entire health system and not just at the clinical/patient interface to explore whether the health system was appropriately focused on SDM and clinicians and patients were fully equipped with all of the information and tools to make a fully informed decision based on the best available evidence. We also acknowledged the local policy imperative to encourage more home dialysis when considered an appropriate option for patients and their families. Frequent faceto-face data analysis and interpretation meetings were convened with team members, key stakeholders and PPI representatives, during which different perspectives were explored and challenged until a consensus was agreed. The researchers who collected data were not known to patients and family members. The researchers were known by many professionals.

\section{Findings}

We present the findings mapped against the adapted MAGIC framework with a focus on the factors that create barriers and enablers to people choosing a home-based treatment from the patient, family member and healthcare professionals' perspective. Further details including additional illustrative quotes are presented in online supplemental appendix 5 .

\section{Prior preferences}

Patients' perspectives

Many people with CKD that we spoke to were asymptomatic and many with symptoms did not always attribute it to their kidney disease. Apathy to disease condition often meant any prior (informal, formal) conversations had little impact on final treatment decisions. Real-life lived experiences impacted on perception of treatment burden, but many people initially catastrophised future treatment, this was especially the case with dialysis.

We found a wide variation in patient knowledge of their own kidney disease, prognosis and potential future treatments and what this might mean for them prior to them commencing the SDM pathway. How and when people recalled that they were told they required treatment varied considerably. We frequently found that a

\section{Table 3 Professional interviews further details}

Topic guide Online supplemental appendix 4 outlines the professional interview content at a high level and includes a topic guide and tools in the form of case studies to help unpick bias codeveloped with PPI input.

Location Place of work for healthcare professionals to fit in with clinical commitments and existing meeting schedules, whereby professionals met in clusters.

Number of Four semistructured interviews individually, two small group interviews with professionals in the same role (eg, interviews consultants, pre-dialysis nurses) and three whole team focus groups.

PPI, patient and public involvement. 
previous legitimate experience such as a family member having dialysis influenced initial preferences. More often it was outdated or inaccurate information picked up from informal sources such as social media or an individual perspective, fear of the unknown, irrational concerns of the magnitude of treatment burden or that dialysis would transform them into being a burden that was highly influential on their initial preferences towards UHD. People who started dialysis in an emergency obviously had no prior preferences, but conversations about switching to a home-based option were met negatively. Most people's initial image of dialysis was of dialysis in a hospital.

\section{Family perspectives}

As with people with CKD, family members often had a previous experience leading to a bias to UHD and most assumed that dialysis only happened in a hospital. On the whole, the family perspective was lacking at these early stages. Many factors influenced this, the person with CKD not wanting to worry the family, they had not been involved in routine clinics or the realities of kidney failure were also not well understood by the family.

\section{Healthcare professionals' perspectives}

Despite many professionals' enthusiasm for home-based treatments, there were inconsistencies, and not all professionals viewed the high number of people on UHD as a problem. Those who were trying to improve access to home dialysis cited an embedded culture, lack of motivation for change fuelled by staffing pressure as key barriers. Healthcare professionals acknowledged a hierarchy in treatments (transplant, home, UHD) in their protocols but in reality expressed challenges in 'delivering the home part consistently across the service' (consultant).

\section{Choice talk-highlighting that reasonable options exist Patient perspectives}

Often the number of choices combined with the ways they were sometimes presented became a barrier (rather than a facilitator) to people initially choosing home. Information days, meeting other patients and peer to peer support were mostly viewed as valuable and reassuring but not necessarily helpful in deciding a future treatment option-especially home dialysis. UHD was the treatment all people initially recognised. This combined with feeling overwhelmed, confused and with little or no opportunities to follow-up their concerns led many to exclude homebased options, often almost immediately. We found that many people's initial discussions about future treatment were not retained to long-term memory. This included discussions over a longer time and special visits designed specifically to discuss all available treatments. Even when starting discussions for future treatments, many patients struggled to modify their behaviours and proactively engage in SDM. Many requested more personalised information and support led by healthcare professionals. Visual graphs, self- monitoring apps, opportunities to ask questions and confidence in sharing their expectations, goals and values were common features in experiences, leading to choosing home dialysis.

\section{Family perspectives}

We frequently found family members taking a back step at initial discussions about future treatments-preferring to push the patient forward as the person the conversations were about. At the same time, family members reported chronic anxiety and frustration at their partners 'for not taking things seriously' (wife of patient). On the whole, the family member had done more reading and internet searches than the person with CKD at this stage. Those with younger families had started to consider impacts on children and what this might mean for them but had not spoken to anybody about it, including their partner. Throughout families tended to worry more than people with CKD. This led to a significant bias from the outset for UHD as it was almost always viewed as the safer option for their relative and for them.

\section{Healthcare professionals' perspectives}

Professionals cited good rapport and drip feeding of information as critical to starting good SDM. At the same time, professionals reported difficulty in predicting disease progression as a key barrier to starting conversations. Many professionals suggested that current protocols in presenting initial options were unhelpful and that they were seeking to change how choices are initially presented. Many reported ongoing frustration with trying to get their patients to accept their disease and engage in more healthy behaviours (which may delay need for future treatment). They also reported health system issues such as short clinic times, inconsistent messaging, lack of research evidence on best dialysis options and lack of up to date communication training for key staff as barriers to starting conversations leading to good SDM. People who started UHD with little or no warning quickly integrated the routine of the dialysis unit as the norm and altering their views towards a home-based dialysis was highly challenging for healthcare professionals.

Some professionals said they wanted to promote more home-based options and avoid mention of UHD in the conversation but felt they had to discuss everything to avoid potential repercussions of not presenting all available options and/or accusations of bias.

\section{Options talk—detailed discussions of pros and cons Patient perspectives}

Very few people distinguished any difference in treatment in terms of health or lifestyle benefits at this stage- they viewed all options as the same. Most people expressed that they wanted their treatment to fit around them and their lifestyle (often cited as the main benefit of a home-based treatment), but this meant different things to different people. Many people said that UHD was the ideal in this respect as they "could drop in on the way home from work, go on the machine and pick up something for dinner on the way home, and have four free days then' (patient). 
Fears over self-administering treatment leading to disfigurement, infections and social isolation were also cited as key barriers for home-based treatment.

\section{Family perspectives}

Many family members in particular spouse/partners had anxieties about their potential responsibilities and their potential changed/or changing role into a carer although this was not always initially recognised by them. Even in cases where patients had expressed a preference for home, family members expressed concerns that they would not have been able to manage especially if something went wrong. In the majority of these cases, patients put their own views aside and went with the preferred option for the family. We frequently saw families identify home dialysis as 'something else for them to do' (wife of patient) and viewed UHD as welcomed respite. However, this time was often taken up with general housekeeping and planning around patients additional healthcare needs. Although some patients felt the same it was mostly the family members who could not reconcile the vision of 'turning their house into a hospital' as well as the wish to separate dialysis from the home and home life.

\section{Healthcare professionals' perspectives}

Despite many professionals' enthusiasm for home-based therapies, they reported ongoing challenges with translating this into practice. They often cited time with patients as a barrier, but when discussed in detail, this was more often coupled with other health system issues such as delays in home dialysis training, staff training, changes of mind not being picked up or actioned on, the role and function of the MDT, comorbidities leading their patients onto complex care pathways of which they have little control over. Some professionals told us that they lacked the necessary in-depth knowledge of specific home therapies to offer clarity and further support.

Some professionals shared how challenging it was to get people to come to terms with and understand the benefits of longer times on dialysis. 'The world is speeding up everything is getting quicker and we expect things nearly instantly these days. Unfortunately evidence shows that slower, gentler dialysis is better but we really struggle to get people to see that and when you have this " 4 hour option' outside the door...' (consultant).

Nobody considered costs when discussing different options. Cost and cost-effectiveness (from any perspective) was never a consideration when having discussions about available treatment options with patients and their families.

\section{Decision talk-making the decision \\ Patient perspectives}

While some people described one main reason for their choice of treatment, others could not articulate clearly why they had opted for one treatment over another. Some people with CKD described a gradual process of decisionmaking and reflected on the benefit of being able to make their choice over a period of time. Others said that the decision was made in one conversation but could rarely articulate the benefits of their decision or the harms of the options that they had discounted. A high number of people felt unable to make a final decision, despite having conversations for months or even years with their healthcare professionals. Many people had not yet even adjusted to the fact that they would need kidney replacement treatment and reported unmet psychological and social care needs. These needs were observed to create barriers to decision-making as they were often perceived as the most urgent or pressing demands that needed addressing before a decision could be made (such as the suitability of their housing or the lack of social support). It was also not uncommon for people to change their mind from a home-based therapy to UHD at this stage. Defaulting to UHD was the easiest option with perceived minimal upheaval at an especially distressing time.

\section{Family perspectives}

Likewise, we observed family members with unmet psychological or social care needs, resulting from their anxieties associated with their relative going into kidney failure. Financial pressures and welfare concerns were the most frequently cited and again this led to a diversion from making (or being involved in) decisions. This in turn led to a high preference for UHD to ease immediate burdens, avoid home adjustments while ensuring that their relatives' health was being looked after. This also gave the impression of freeing up time to adjust for current and future changing circumstance.

\section{Healthcare professionals' perspectives}

Some professionals commented on health systems and processes undermining or undoing their SDM. 'So, I can council my patients for years, we get to know them and have detailed discussions, overtime they are all prepped and ready for $P D$, then they go off to the multi-disciplinary team or to start preparations etc and the next time I see them they are on the unit. It is one thing to say shared decision-making another thing entirely to actually make it work' (consultant). Often professionals were asked at this stage, 'what would you do, which treatment would you go for' (pre-dialysis specialist nurse) and it was something many felt challenging to manage. Healthcare professionals also reported frustrations trying to explain to people the urgent need to make a decision and engage with those who were proactively delaying treatment.

There were inconsistencies with commissioners and patient perspectives on home-based treatments throughout the pathway. The increasing number of options were consistently marketed as a triumph, 'homebased dialysis is brilliant we can fit it to anybody's needs, they get longer better dialysis and so will feel better and live longer. It's cheaper for the NHS, for the patient and family as they have the freedom to live the life they want to lead, what's not to love!' (correspondence with service commissioners). However, when given the choice, people were still defaulting to a standard treatment and very few 
people discussed their decision as a 'no-brainer for home' (correspondence with PPIs not in the Welsh system). Even people who had chosen home-based treatment still expressed doubt and visible concerns about their decision and uncertainty about the future.

The perceived safety of UHD, the respite offered by dialysis free days and the capacity of their staff to act as surrogate families as well as social hubs made implementing pre-dialysis SDM exceptionally difficult for healthcare professionals. Health system issues (as well as the health of the person) meant that what people initially chose was not necessarily where they ended up.

\section{Logic model stages, moderators and outcomes of getting onto a home therapy and road map development with recommendations}

The above findings were presented at a wider PPI meeting (box 1), where quotes were mapped alongside the threetalk model (figure 1). Facilitated discussion identified six tangible stages of the SDM pathway, with moderators and outcomes, which was refined and presented as a logic model (table 4) and these were mapped alongside the identified barriers and facilitators from the multiple perspectives in the interviews. At this stage, the logic model was refined and shared via email and follow-up consultations with clinical members of the research team, a PPI group and selected members of the MDT (including kidney social workers and psychologists) to highlight what they saw as the intermediate and long-term outcomes for people getting onto home dialysis. The road map outlining service improvement recommendations (table 5) was co-produced alongside the logic model of stages, moderators and outcomes of getting onto a home therapy and aimed to highlight key findings in a structured way with actionable recommendations. The road map and logic model aimed to be applicable to all kidney services regardless of their configuration.

\section{DISCUSSION}

\section{Statement of principal findings}

CKD was not well understood by many people living with kidney disease and their family members. Health systems and various clinical pathways into secondary care made it challenging to implement SDM between patients and professionals as intended. SDM was inconsistent and often too generic to be helpful for patients, family members or professionals. The changed/changing needs of people with CKD or the needs of the family as future treatment plans were developed with professionals were not well understood. The focus on providing (important) but largely clinically focused information missed opportunities to better manage expectations, identify goals and engage patients in good SDM. Family members worried more than patients, and this tended to lead towards a strong bias for UHD. People with CKD and family members defaulted to the perceived simplest option
(UHD), often to pick up their unmet psycho/social care needs.

Complex care pathways meant that what people chose was not necessarily a reflection of where they ended up. Sometimes people appeared to land on a home-based option more by luck than judgement as few people could discern with any real certainty the benefits of their option or crucially the harms of hospital-based dialysis on quality of life, survival and sustainable services. ${ }^{17} 43$

\section{IMPLICATIONS FOR CLINICIANS AND POLICYMAKERS}

Options for home dialysis are increasing. PD and HHD can both be done during the day or overnight. People have the freedom to switch between day and night dialysis and even choose the length of time they dialyse and how many times per week. The benefits of this-cited by so many senior healthcare professionals, commissioners and advocates-are not translating into increases in people on a home therapy.

In terms of implementing SDM across the kidney service, the challenges have been similar to those documented elsewhere, including complex health service configurations, lack of engagement across system levels, confidence from healthcare professionals and general health literacy of the public. ${ }^{44-46}$ In this study, we also observed that SDM was not routinely implemented alongside protocols that explain treatment options in a hierarchy. Therefore, SDM in pre-dialysis services is on trajectory to nothing and like other studies have shown, SDM on its own achieves nothing. ${ }^{47}$ Although no healthcare professionals expressed that they felt UHD was better or that they were biased to UHD very few people saw or expressed the fact that over $80 \%$ of the dialysis population are all on the same type of dialysis as a problem. Healthcare professionals are under pressure, health systems struggle to cope with demand and are increasingly picking up unmet social care needs but unless people across the multiple levels accept the barriers to home dialysis as a problem then SDM (including the various tools, training and support packages) is likely to have little impact.

\section{STRENGTHENS AND WEAKNESSES OF THE STUDY}

This was an in-depth qualitative investigation into SDM in the context of pre-dialysis decision-making, across a country wide diverse population and five kidney services. The study was largescale, informed by theory, used a purposive sampling frame to address known gaps and explored multiple perspectives: patients, family members and healthcare professionals. Data collection was completed before the COVID-19 pandemic. We originally planned to interview a minimum of 30 patients and 30 family members. In reality, we interviewed more patients $^{37}$ and fewer carers ${ }^{19}$ than planned. As Wales is a predominately white population, Black and Minority Ethnic (BAME) perspectives are a noted gap. 
Table 4 Logic model of stages, moderators and outcomes of getting onto a home therapy

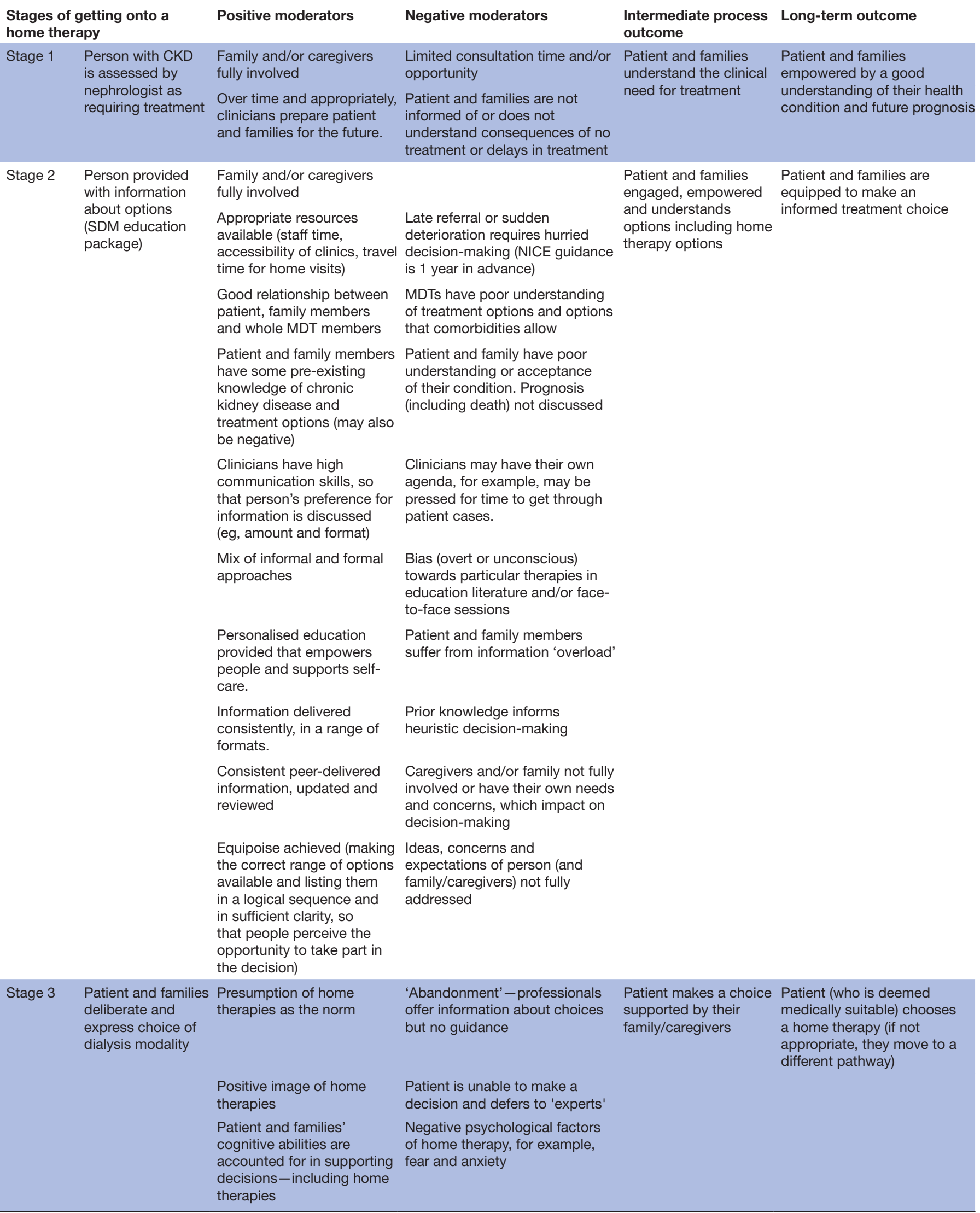


Table 4 Continued

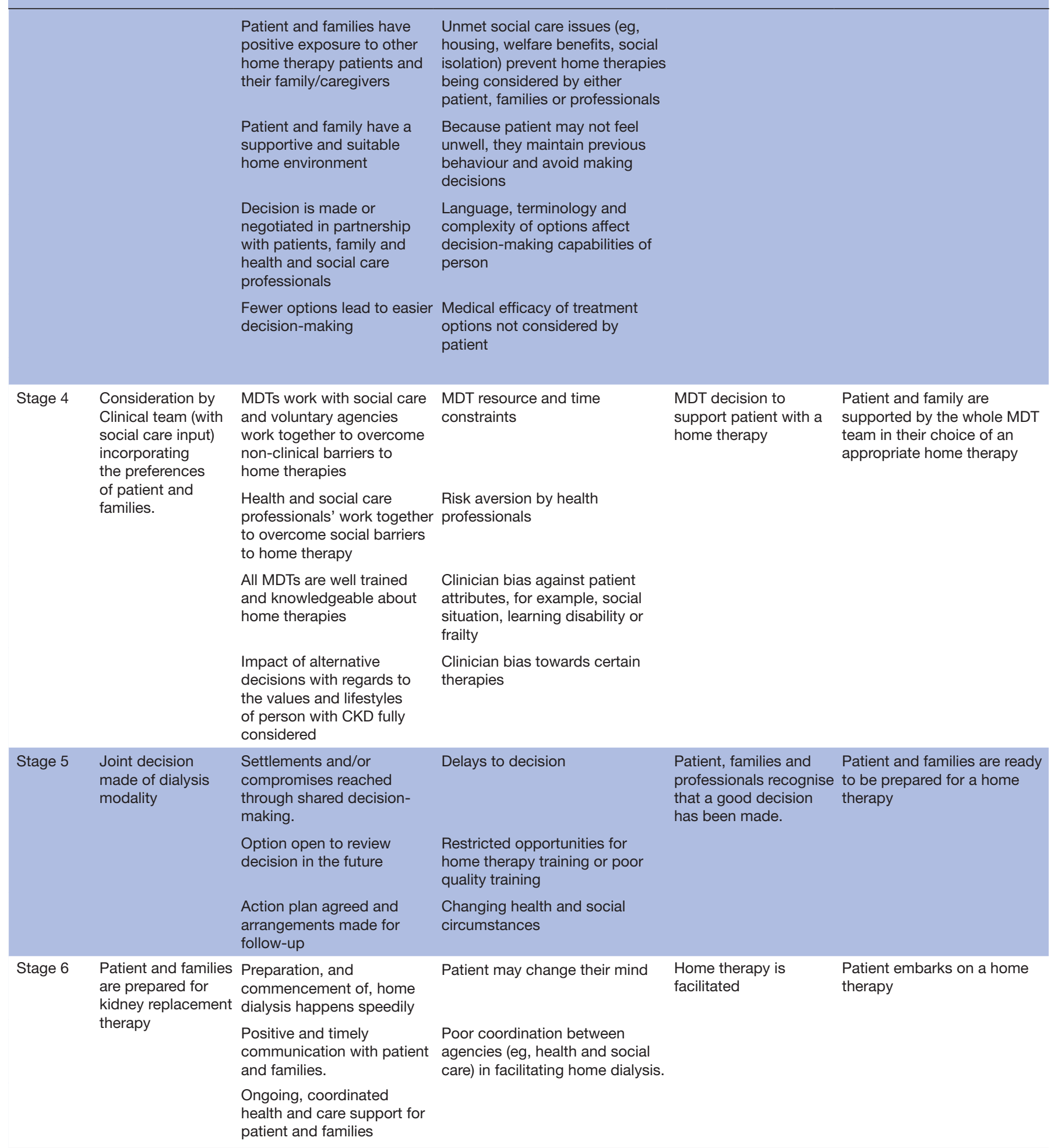

CKD, chronic kidney disease; MDT, Multi-disciplinary team; NICE, National Institute for Health and Care Excellence; SDM, shared decision-making.

\section{Strengths and weaknesses in relation to other studies}

While studies have examined structural issues, general support and patient attitudes to home therapies, ${ }^{4-50}$ the process of SDM in the context of better understanding barriers to home dialysis has not been examined in detail. By framing findings within a recognised model, we show how people with CKD and their family members behave through the decision-making process as well as observing and understanding the perceptions and behaviours of healthcare professionals along the same continuum. Likewise, Tong and colleagues report that the language used with patients about kidney disease is often alien 
Table 5 Road map and recommendations for service delivery change to increase uptake of home dialysis

\begin{tabular}{|c|c|}
\hline 1. & nanagers and commissioners, and reflected in education. \\
\hline 3. & $\begin{array}{l}\text { Redesign education packages. so that patients rely less on unreliable or industry-sponsored resources. This is likely to be a mixture } \\
\text { of online material, group discussion (delivered by peers where possible) and one-to-one sessions. All MDTs have a key role in } \\
\text { identifying which education package is best suited for each patient, but the content should be standardised regardless of how or who } \\
\text { delivers it. Education packages should also fully engage the family in recognition of their crucial role in decision-making. }\end{array}$ \\
\hline 4. & $\begin{array}{l}\text { Encourage patient engagement and activation at an early stage rather than wait until the time of decision-making and then expecting } \\
\text { people to become active in their own care. For example, two-way patient portals, which both deliver education/information updates/ } \\
\text { patient results/clinic letters to the patient and also let the patient upload information, which is important to their care (eg, blood } \\
\text { pressure/weight/key symptoms) and viewed by patients. }\end{array}$ \\
\hline 7. & $\begin{array}{l}\text { Social and psychological support. A high burden of anxiety (and likely depression) that is unrecognised by clinical teams will impact } \\
\text { on decision-making. There are tools that clinicians could use to aid in identifying these symptoms (eg, validated questionnaires); they } \\
\text { need to draw on specialist kidney social work and psychologist capacity; and be able to sign-post to appropriate external support. }\end{array}$ \\
\hline
\end{tabular}

CKD, chronic kidney disease; eGFR, Estimated Glomeruler Filtration Rate; MDT, Multi-disciplinary team.

and frightening, and the barriers to home dialysis are complex. ${ }^{51} 52$ This study adds more by highlighting the different experiences and perspectives of SDM and barriers to home dialysis. Sometimes, different perspectives expressed near identical views and frustrations, for example, family members and professionals both reported similar challenges engaging patients to take their kidney disease seriously, engage in SDM and generally in more healthy behaviours. This suggests that the family could potentially be reconfigured as a facilitator to improve uptake of home dialysis if they were more proactively involved and their unmet needs were addressed.

\section{Suggestions for future research and unanswered questions}

A new health system wide reorganisation supported by a comprehensive overhaul of patient education materials needs to be developed and implemented to ensure consistent practice across Wales supplemented by a trial to examine its effectiveness. Any such trial would need to be a step wedge design with gradual introduction of the new system and associated enhancements into each subsequent dialysis service. This will be an important next step as although many SDM tools and guidance are in operation (and more are constantly being produced), few have been robustly tested at a system level, from the multiple perspectives and alongside specific service change targets. NICE has recently updated their SDM guidance and is collating existing SDM interventions. ${ }^{53}$ Future research should take account of these developing resources to ensure consistency, translatability, shared goals and evidence-based practices are developed and adhered to.

\section{CONCLUSION}

People with CKD are not being adequately prepared or educated about the most appropriate treatment options for them or the benefits, harms, outcomes or costs of the different options. Patients and family members are too reliant on information or misconceptions gained from sources with varying accuracy and credibility. Healthcare professionals lack the skills to individually tailor the decision-making process for each person and some are not familiar with using shared decision models to inform their conversations with patients. Patients and professionals need greater awareness of the relative costs and benefits of different treatment options to inform their SDM processes.

Study findings are important as they highlight the critical points in the decision-making processes, where changes to practice can potentially bring about better SDM and outcomes with patients. People with CKD need to be better prepared and their preconceived ideas and misconceptions gently challenged. Healthcare professionals need to update their knowledge on contemporary kidney therapies in order to provide the best advice and guidance. Healthcare practitioners can use the logic model and road map to clinical change to benchmark and improve their own practice.

Twitter Jane Noyes @janenoyes 
Acknowledgements The authors gratefully acknowledge the participation of people with kidney disease, their families and caregivers, and all the healthcare professionals who participated in the study. The authors also wish to acknowledge the wider network of public and professionals who helped to coproduce the study through the Wales Kidney Research Unit. We acknowledge the important role of Teri Howells, a kidney patient representative in the research team who sadly died during the study. We are further grateful to David Fellowes who volunteered to take her place. Both Teri and David were invaluable members of the team providing advice, feedback on findings and reviewing drafts of the protocol (Teri) and subsequent papers (David). The authors acknowledge contributions made by Barbara Neukirchinger who undertook coding of transcripts, synthesised data and provided summaries of data and David Dallimore who supported operationalisation of the study procedures, collected data, undertook coding, provided summaries and supported the overall conduct of the study. David Dallimore also developed an initial draft of the paper that was subsequently evolved over time by the authors. This work was funded by Health and Care Research Wales (HCRW). The views expressed are those of the authors and not necessarily those of NHS Wales and HCRW.

Contributors $\mathrm{JN}$ is guarantor of the paper. She was significantly involved in the conception and design of the research, oversight of data collection and analysis, drafting and revisions of the manuscript and signing off the final version of the manuscript. GR was significantly involved in the conception and design of the research, interpreting the data, and was a major contributor in writing and signing off the final version of the manuscript. GW was significantly involved in the conception and design of the research, was involved in acquiring and interpreting the data, and made a contribution to revisions of the manuscript. JC was significantly involved in the conception and design of the research, interpreting the data and made a contribution to revisions and signing off the final version of the manuscript of the manuscript. LML was involved in the conception and design of the research, acquired, analysed and interpreted the data, supported drafting and signing off the final version of the manuscript.

Funding This work was funded by Health and Care Research Wales (HCRW). Funder number RfPPB17-1423(T). The views expressed are those of the authors and not necessarily those of NHS Wales and HCRW.

Competing interests None declared.

Patient consent for publication Not applicable.

Ethics approval All participants—patients, family and professionals—were given detailed written information on the study and were asked to sign a consent form indicating their willingness to participate. Anonymity was assured to all participants. Participants retained the right at all times to withdraw from the study at any time patients were assured that withdrawal would have no impact on their current or future treatment. Ethical approval for this study was granted by the Wales Research Ethics Committee (Reference 19/WA/0020).

Provenance and peer review Not commissioned; externally peer reviewed.

Data availability statement Data are available upon reasonable request. All data relevant to the study are included in the article or uploaded as supplementary information.

Supplemental material This content has been supplied by the author(s). It has not been vetted by BMJ Publishing Group Limited (BMJ) and may not have been peer-reviewed. Any opinions or recommendations discussed are solely those of the author(s) and are not endorsed by BMJ. BMJ disclaims all liability and responsibility arising from any reliance placed on the content. Where the content includes any translated material, BMJ does not warrant the accuracy and reliability of the translations (including but not limited to local regulations, clinical guidelines, terminology, drug names and drug dosages), and is not responsible for any error and/or omissions arising from translation and adaptation or otherwise.

Open access This is an open access article distributed in accordance with the Creative Commons Attribution Non Commercial (CC BY-NC 4.0) license, which permits others to distribute, remix, adapt, build upon this work non-commercially, and license their derivative works on different terms, provided the original work is properly cited, appropriate credit is given, any changes made indicated, and the use is non-commercial. See: http://creativecommons.org/licenses/by-nc/4.0/.

\section{ORCID iDs}

Jane Noyes http://orcid.org/0000-0003-4238-5984

Gareth Roberts http://orcid.org/0000-0003-4496-6539

James Chess http://orcid.org/0000-0001-8805-6962

Leah Mc Laughlin http://orcid.org/0000-0003-0185-6639
REFERENCES

1 Renal services delivery plan: 2016 to 2020 (WHC/2016/042) | GOV. WALES. Available: https://gov.wales/renal-services-delivery-plan2016-2020-whc2016042 [Accessed 06 Sep 2021].

2 Jansz TT, Noordzij M, Kramer A, et al. Survival of patients treated with extended-hours haemodialysis in Europe: an analysis of the ERA-EDTA registry. Nephrol Dial Transplant 2020;35:488-95.

3 McDonald SP, Marshall MR, Johnson DW, et al. Relationship between dialysis modality and mortality. J Am Soc Nephrol 2009;20:155-63.

4 Sarafidis P, Faitatzidou D, Papagianni A. Benefits and risks of frequent or longer haemodialysis: weighing the evidence. Nephrol Dial Transplant 2021;36:1168-76.

5 Komenda P, Gavaghan MB, Garfield SS, et al. An economic assessment model for in-center, conventional home, and more frequent home hemodialysis. Kidney Int 2012;81:307-13.

6 Chertow GM, Levin NW, Beck GJ, et al. Long-term effects of frequent in-center hemodialysis. J Am Soc Nephrol 2016;27:1830-6.

7 Walker RC, Howard K, Morton RL. Home hemodialysis: a comprehensive review of patient-centered and economic considerations. Clinicoecon Outcomes Res 2017;9:149-61.

8 Wyld M, Morton RL, Hayen A, et al. A systematic review and metaanalysis of utility-based quality of life in chronic kidney disease treatments. PLoS Med 2012;9:e1001307.

9 Walker RC, Howard K, Morton RL, et al. Patient and caregiver values, beliefs and experiences when considering home dialysis as a treatment option: a semi-structured interview study. Nephrol Dial Transplant 2016;31:133-41.

10 Walker RC, Howard K, Tong A, et al. The economic considerations of patients and caregivers in choice of dialysis modality. Hemodial Int 2016;20:634-42.

11 Treharne C, Liu FX, Arici M, et al. Peritoneal dialysis and in-centre haemodialysis: a cost-utility analysis from a UK payer perspective. Appl Health Econ Health Policy 2014;12:409-20 https://pubmed. ncbi.nlm.nih.gov/25017433/

12 Beaudry A, Ferguson TW, Rigatto C, Dumanski K, et al. Cost of dialysis therapy by modality in Manitoba. Clin J Am Soc Nephrol 2018;13:1197-203.

13 Harwood L, Clark AM. Understanding pre-dialysis modality decisionmaking: a meta-synthesis of qualitative studies. Int J Nurs Stud 2013;50:109-20.

14 Majeed-Ariss R, Jayanti A, Schulz T, Schulz, Wearden M, et al. The anticipated and the lived experience of home and incentre haemodialysis: is there a disconnect? J Health Psychol 2017;22:1524-33.

15 Elwyn G, Frosch D, Thomson R, et al. Shared decision making: a model for clinical practice. J Gen Intern Med 2012;27:1361-7.

16 National Institute for Health and Care Excellence (NICE). Renal research recommendations. Available: https://www. nice.org.uk/search?om=[\%7B\%22ndt\%22:[\%22Research recommendations \%22]\%7D]\&ps=50\&q=renal\&sp=on [Accessed 21 Oct 2019].

17 Himmelfarb J, Vanholder R, Mehrotra R, et al. The current and future landscape of dialysis. Nat Rev Nephrol 2020;16:573-85 https://www. nature.com/articles/s41581-020-0315-4

18 UK renal registry. Audit portal. Available: https://www.renalreg.org/ datasets/audit-portal/ [Accessed 11 Oct 2019].

19 Combes G, Sein K, Allen K. How does pre-dialysis education need to change? Findings from a qualitative study with staff and patients. BMC Nephrol 2017;18:1-11.

20 Ladin K, Lin N, Hahn E, et al. Engagement in decision-making and patient satisfaction: a qualitative study of older patients' perceptions of dialysis initiation and modality decisions. Nephrol Dial Transplant 2017;32:1394-401.

21 NICE. Bringing the benefits of home haemodialysis home. Available: https://www.nice.org.uk/sharedlearning/bringing-the-benefits-ofhome-haemodialysis-home [Accessed 06 Sep 2021].

22 Saran R, Robinson B, Abbott KC, et al. Us renal data system 2017 annual data report: epidemiology of kidney disease in the United States. Am J Kidney Dis 2018;71:A7.

23 Mclaughlin L. Assessing the efficacy of co-production to better understand the barriers to achieving sustainability in NHS chronic kidney services and create alternate pathways. BMJ Open 2021.

24 McLaughlin K, Manns B, Mortis G, et al. Why patients with ESRD do not select self-care dialysis as a treatment option. Am J Kidney Dis 2003;41:380-5.

25 Agar JW, Perkins A, Heaf JG. Home hemodialysis: infrastructure, water, and machines in the home. Hemodial Int 2015;19 Suppl 1:S93-111.

26 Zhang A-H, Bargman JM, Lok CE, et al. Dialysis modality choices among chronic kidney disease patients: identifying the gaps 
to support patients on home-based therapies. Int Urol Nephrol 2010;42:759-64.

27 Baillie J, Lankshear A. Patients' and relatives' experiences of peritonitis when using peritoneal dialysis. J Ren Care 2015;41:177-86

28 Baillie J, Lankshear A. Patient and family perspectives on peritoneal dialysis at home: findings from an ethnographic study. J Clin Nurs 2015;24:222-34.

29 Roberts G, Chess JA, Howells T, et al. Which factors determine treatment choices in patients with advanced kidney failure? A protocol for a co-productive, mixed methods study. BMJ Open 2019;9:e031515.

30 Bryman A, GBurgess R. Analyzing qualitative data. Routledge, 1994.

31 Booth A, Hannes K, Harden A. COREQ (consolidated criteria for reporting qualitative studies). Guidel Report Heal Res A User's Man 2014:214-26.

32 Joseph-Williams N, Lloyd A, Edwards A, et al. Implementing shared decision making in the NHS: lessons from the magic programme. BMJ 2017;357:j1744.

33 Joseph-Williams N, Williams D, Wood F, et al. A descriptive model of shared decision making derived from routine implementation in clinical practice ('implement-SDM'): qualitative study. Patient Educ Couns 2019:102:1774-85.

34 Elwyn G, Durand MA, Song J, et al. A three-talk model for shared decision making: multistage consultation process. BMJ 2017;359:4891:j4891.

35 MacNeill SJ, Ford D. Uk renal registry 19th annual report: chapter 2 UK renal replacement therapy prevalence in 2015: national and centre-specific analyses. Nephron 2017;137 Suppl 1:45-72.

36 Measuring the health and well-being of a nation public health outcomes framework for Wales 2016.

37 Boonstra MD, Reijneveld SA, Foitzik EM. How to tackle health literacy problems in chronic kidney disease patients? A systematic review to identify promising intervention targets and strategies. Nephrol Dial Transplant 2020;36. doi:10.1093/ndt/gfaa273. [Epub ahead of print: 22 Dec 2020].

38 NIHR Central Commissioning Facility, Patient and Public Involvement Team NCCF. UK standards for public involvement, 2019. Available: https://sites.google.com/nihr.ac.uk/pi-standards/home [Accessed 19 Feb 2020].

39 Revenson TA, Griva K, Luszczynska A. Caregiving in the illness context. Springer, 2016: 1-156.
40 Ritchie J, Lewis J. Qualitative research practice : a guide for social science students and researchers. Sage Publications, 1980: 336.

41 Lincoln YS, Guba EG, Pilotta JJ. Naturalistic inquiry. Beverly Hills Calif: Sage Publications, 1985: 9. 438-9.

42 A healthier wales: long term plan for health and social care | GOV. WALES. Available: https://gov.wales/healthier-wales-long-term-planhealth-and-social-care [Accessed 06 Sep 2021].

43 The weekly break from dialysis is harmful to patients with kidney failure, 2020. Available: https://evidence.nihr.ac.uk/alert/the-weeklybreak-from-dialysis-is-harmful-to-patients-with-kidney-failure/ [Accessed 13 May 2021].

44 Alsulamy N, Lee A, Thokala P, et al. What influences the implementation of shared decision making: an umbrella review. Patient Educ Couns 2020;103:2400-7.

45 Pel-Littel RE, Snaterse M, Teppich NM. Barriers and facilitators for shared decision making in older patients with multiple chronic conditions: a systematic review [Internet]. Vol. 21, BMC Geriatrics. BioMed Central Ltd, 2021: 1-14.

46 Finlay J, Wilson T, Javaheri PA, et al. Patient and physician perspectives on shared decision-making for coronary procedures in people with chronic kidney disease: a patient-oriented qualitative study. CMAJ Open 2020;8:E860-8 /pmc/articles/ PMC7867031/

47 Elwyn G. Expecting too much of "patient decision aids". BMJ, 2019.

48 Ladin K, Pandya R, Kannam A, et al. Discussing conservative management with older patients with CKD: an interview study of nephrologists. Am J Kidney Dis 2018;71:627-35

49 Schell JO, Green JA, Tulsky JA, et al. Communication skills training for dialysis decision-making and end-of-life care in nephrology. Clin $J$ Am Soc Nephrol 2013;8:675-80.

50 Berger JR, Hedayati SS. Renal replacement therapy in the elderly population. Clin J Am Soc Nephrol 2012;7:1039-46

51 Walker RC, Morton RL, Palmer SC, et al. A discrete choice study of patient preferences for dialysis modalities. Clin J Am Soc Nephrol 2018;13:100-8.

52 James LJ, Wong G, Tong A, et al. Discrete choice experiments to elicit patient preferences for decision making in transplantation. Transplantation 2021;105:960-7 https://pubmed.ncbi.nlm.nih.gov/ 33093407/

53 Carmona C, Crutwell J, Burnham M, et al. Shared decision-making: summary of NICE guidance. BMJ 2021;373:n1430. 